\title{
ON HOLOMORPHIC REGULARIZATION OF STRONGLY NONLINEAR SINGULARLY PERTURBED PROBLEMS
}

\author{
V.I. KACHALOV
}

\begin{abstract}
The method of holomorphic regularization, being a logical continuation of the method of S.A. Lomova, allows one to construct solutions to nonlinear singularly perturbed initial problems as series in powers of a small parameter converging in the usual sense. The method is based on a generalization of the Poincare decomposition theorem: in the regular case, solutions depend holomorphically on a small parameter, in the singular case the first integrals inherit this dependence. Having arised in the framework of the regularization method, S.A. Lomov's concept of a pseudo-analytic (pseudo-holomorphic) solution of singularly perturbed problems initiated the formation of the analytic theory of singular perturbations. This theory is designed to equalize the rights of regular and singular theories. In the first case, under sufficiently general assumptions, the series obtained in the solution of problems in powers of the small parameter converge in the usual sense, and in the second case they are basically asymptotic. A vivid example of the holomorphic dependence on a parameter of the solution to a differential equation is given by Poincare's decomposition theorem.

In the present paper, the holomorphic regularization method is applied for constructing pseudo-holomorphic solutions to a singularly perturbed first order equation and to a second order Tikhonov system.
\end{abstract}

Keywords: holomorphic regularization, commutation relation, pseudo-holomorphic solution, Tikhonov system, passage to the limit.

Mathematics Subject Classifcation: 34K26

\section{INTRODUCTION}

Lomov's regularization method [2], 3] allows one to construct solutions of singularly perturbed problems as power series in a small parameter converging not only asymptotically but also in the usual sense. Such solutions are called pseudo-analytic (pseudo-holomorphic) and in this way, Poincaré theorem on expansion in parameter is developed also in the theory of differential equations.

\section{HolOMORPHIC IN PARAMETER FIRST INTEGRALS OF SINGULARLY PERTURBED} EQUATIONS

We consider the Cauchy problem

$$
\varepsilon \frac{d w}{d z}=f(z, w), \quad w\left(z_{0}, \varepsilon\right)=w_{0}
$$

in some domain $\Omega_{0}$ of complex variables $z$ and $w$ containing the initial point $P_{0}\left(z_{0}, w_{0}\right)$. In a regular case, that is, as a small complex parameter $\varepsilon$ is involved holomorphically in the

V.I. KACHALOV, ON HOLOMORPHIC REGULARIZATION OF STRONGLY NONLINEAR SINGULARLY PERTURBED PROBLEMS.

(C) KaChaLOV V.I. 2018.

Submitted May 29, 201\%. 
right hand of the equation, according Poincaré theorem, the equation possesses a solution holomorphic at the point $\varepsilon=0$ and satisfying the given initial condition. It is clear that for equation (1) this is not necessarily the case for a complicated right hand side since as $\varepsilon=0$, this equation is no longer differential. Nevertheless, there exists a characteristics inheriting a holomorphic (and even entire) dependence of the left hand side in equation (1) on the small parameter.

We introduce some notations. Let $\mathcal{A}_{z_{0}}$ be the algebra of functions of one complex variable $z$ holomorphic at the point $z_{0}$, and $\mathcal{A}_{z_{0} w_{0}}$ be the algebra of functions of two complex variables holomorphic at the point $P_{0}\left(z_{0}, w_{0}\right)$.

Theorem 1. Assume that the function $f(z, w)$ is holomorphic in $\Omega_{0}$ and does not vanish. Then the mappings defined by the formula

$$
\begin{aligned}
H_{f}^{\varepsilon}[\varphi(z)] \equiv \varphi(z) & -\varepsilon \int_{w_{0}}^{w} \frac{\varphi^{\prime}(z) d w_{1}}{f\left(z, w_{1}\right)}+\varepsilon^{2} \int_{w_{0}}^{w}\left(\frac{\partial}{\partial z} \int_{w_{0}}^{w_{1}} \frac{\varphi^{\prime}(z) d w_{2}}{f\left(z, w_{2}\right)}\right) \frac{d w_{1}}{f\left(z, w_{1}\right)} \\
& -\varepsilon^{3} \int_{w_{0}}^{w}\left(\frac{\partial}{\partial z} \int_{w_{0}}^{w_{1}}\left(\frac{\partial}{\partial z} \int_{w_{0}}^{w_{2}} \frac{\varphi^{\prime}(z) d w_{3}}{f\left(z, w_{3}\right)}\right) \frac{d w_{2}}{f\left(z, w_{2}\right)}\right) \frac{d w_{1}}{f\left(z, w_{1}\right)}+\ldots
\end{aligned}
$$

form a holomorphic at the point $\varepsilon=0$ family $\left\{H_{f}^{\varepsilon}\right\}$ of continuous homomorphisms of the algebra $\mathcal{A}_{z_{0}}$ into the algebra $\mathcal{A}_{z_{0} w_{0}}$ and the commutation relation

$$
H_{f}^{\varepsilon}[\varphi(z)]=\varphi\left(H_{f}^{\varepsilon}[z]\right), \quad \varphi(z) \in \mathcal{A}_{z_{0}},
$$

is satisfied. The image of this family consists of the first integrals $U_{\varphi}(z, w, \varepsilon)=H_{f}^{\varepsilon}[\varphi(z)]$ of equation (1) holomorphic in the small parameter $\varepsilon$.

The proof of Theorem 1 is based on the Cauchy integral formula for functions of several complex variables and general properties of the first integrals for differential equations [5].

\section{PSEUdoholomorphiC SOlUtions TO SINGULARLY PERTURBED PROBLEMS}

Definition 1. A solution $w(z, \varepsilon)$ to Cauchy problem (1) is called pseudo-holomorphic at the point $\varepsilon=0$ if there exists a function $W(z, \eta, \varepsilon)$ holomorphic at the point $Q\left(z_{0}, 0,0\right)$ in the space of complex variables $(z, \eta, \varepsilon)$ such that for each $\varepsilon$ in some neighbourhood of the point $\varepsilon=0$ there exists a neighbourhood $\omega_{z}^{\varepsilon}$ of the point $z_{0}$ such that the identity holds:

$$
w(z, \varepsilon)=W\left(z, \frac{\varphi(z)}{\varepsilon}, \varepsilon\right),
$$

where $\varphi(z)$ is some function $\mathcal{A}_{z_{0}}$ satisfying the condition $\varphi\left(z_{0}\right)=0$.

If the series

$$
\sum_{n=0}^{\infty} W_{n}(z, \eta) \varepsilon^{n}
$$

representing the function $W(z, \eta, \varepsilon)$ converges uniformly in $z$ on each compact set $T_{z_{0}}$ containing the point $z_{0}$ for each $\eta$ in an unbounded connected set $G$ in the complex plane of the variable $\eta$ and the convergence holds in some neighbourhood (depending on $\eta$ ) of the point $\varepsilon=0$, then the solution $w(z, \varepsilon)$ is called pseudo-holomorphic in the global sense.

We dwell on a more important, in our opinion, global pseudo-holomorphy. 
Theorem 2. If the equation

$$
\varphi^{\prime}(z) \int_{w_{0}}^{w} \frac{d w_{1}}{f\left(z, w_{1}\right)}=\eta
$$

is solvable with respect to $w$ for each $\eta$ in an unbounded simply-connected domain $G \subset \mathbb{C}_{\eta}$ and its solution $w=W_{0}(z, \eta)$ is bounded on a set $T_{z_{0}} \times G$, where $T_{z_{0}} \subset \mathbb{C}_{z}$ is some compact set containing the point $z_{0}$, then the solution $w(z, \varepsilon)$ to initial problem (1) is pseudo-holomorphic in the global sense.

Proof. Let $\mathcal{K}$ be a compact set containing the point $\eta=0$ and being a closure of some simplyconnected subdomain of the domain $G$. By the implicit function theorem, for each point $\eta \in \mathcal{K}$ there exists a neighbourhood $\sigma_{\eta}$, in which the equation

$$
V(z, w, \varepsilon)=\eta
$$

where

$$
\begin{aligned}
V(z, w, \varepsilon)= & \varphi^{\prime}(z) \int_{w_{0}}^{w} \frac{d w_{1}}{f\left(z, w_{1}\right)}-\varepsilon \int_{w_{0}}^{w}\left(\frac{\partial}{\partial z} \int_{w_{0}}^{w_{1}} \frac{\varphi^{\prime}(z) d w_{2}}{f\left(z, w_{2}\right)}\right) \frac{d w_{1}}{f\left(z, w_{1}\right)} \\
& +\varepsilon^{2} \int_{w_{0}}^{w}\left(\frac{\partial}{\partial z} \int_{w_{0}}^{w_{1}}\left(\frac{\partial}{\partial z} \int_{w_{0}}^{w_{2}} \frac{\varphi^{\prime}(z) d w_{3}}{f\left(z, w_{3}\right)}\right) \frac{d w_{2}}{f\left(z, w_{2}\right)}\right) \frac{d w_{1}}{f\left(z, w_{1}\right)}-\ldots,
\end{aligned}
$$

has a holomorphic in $\varepsilon$ solution $W(z, \eta, \varepsilon)$ uniformly in $z \in T_{z_{0}}$. In the covering $\left\{\sigma_{\eta}\right\}$ of the compact set $\mathcal{K}$ we choose a finite subcovering $\left\{\sigma_{\eta}\right\}_{1}^{N}$. Then the function $W(z, \eta, \varepsilon)$ is holomorphic uniformly in $z \in T_{z_{0}}$ for each $\eta \in \mathcal{K}$ in the neighbourhood $|\varepsilon|<\varepsilon_{0}$, where $\varepsilon_{0}>0$ is the smallest among such constants associated with the finite subcovering.

Let the parameter $\varepsilon$ in equation (1) satisfies the inequality $0<\varepsilon<\varepsilon_{0}$. By $\widetilde{T}_{z_{0}}$ we denote the set of the points in $T_{z_{0}}$, for which the values $\eta=\varphi(z) / \varepsilon$ belong to $\mathcal{K}$. Then the solution $w(z, \varepsilon)$ can be represented as the series

$$
w(z, \varepsilon)=\sum_{n=0}^{\infty} \varepsilon^{n} W_{n}\left(z, \frac{\varphi(z)}{\varepsilon}\right)
$$

converging in the usual sense in $\widetilde{T}_{z_{0}}$. The proof is complete.

Let us write out the formulae for the leading terms in series (8):

$$
W_{1}=-\left.\frac{V_{1}}{V_{2}}\right|_{w=W_{0}(z, \varphi(z) / \varepsilon)}, \quad W_{2}=-\left.\frac{V_{11} V_{2}^{2}-2 V_{12} V_{1} V_{2}+V_{22} V_{1}^{2}}{2 V_{2}^{2}}\right|_{w=W_{0}(z, \varphi(z) / \varepsilon)},
$$

where

$$
\begin{aligned}
& V_{1}=-\int_{w_{0}}^{w}\left(\frac{\partial}{\partial z} \int_{w_{0}}^{w_{1}} \frac{\varphi^{\prime}(z) d w_{2}}{f\left(z, w_{2}\right)}\right) \frac{d w_{1}}{f\left(z, w_{1}\right)} ; \\
& V_{2}=\frac{\varphi^{\prime}(z)}{f(z, w)} ; \\
& V_{11}=2 \int_{w_{0}}^{w}\left(\frac{\partial}{\partial z} \int_{w_{0}}^{w_{1}}\left(\frac{\partial}{\partial z} \int_{w_{0}}^{w_{2}} \frac{\varphi^{\prime}(z) d w_{3}}{f\left(z, w_{3}\right)}\right) \frac{d w_{2}}{f\left(z, w_{2}\right)}\right) \frac{d w_{1}}{f\left(z, w_{1}\right)} ;
\end{aligned}
$$




$$
\begin{aligned}
& V_{12}=-\frac{1}{f(z, w)}\left(\frac{\partial}{\partial z} \int_{w_{0}}^{w} \frac{\varphi^{\prime}(z) d w_{1}}{f\left(z, w_{1}\right)}\right) ; \\
& V_{22}=-\frac{\varphi^{\prime}(z) f_{w}^{\prime}(z, w)}{f^{2}(z, w)} .
\end{aligned}
$$

In the following examples we assume that $G=(-\infty ; 0], T_{z_{0}}$ is a segment in the real axis, whose left ends is at the point $z_{0}=0$ and $\varepsilon>0$.

Example 1. We consider the Cauchy problem:

$$
\varepsilon \frac{d w}{d z}=w^{2}-e^{2 z}, \quad w(0, \varepsilon)=0 .
$$

The solution constructed by the method of holomorphic regularization is of the form:

$$
w(z, \varepsilon)=e^{z} \tanh \frac{1-e^{z}}{\varepsilon}+\frac{\varepsilon}{2} \tanh ^{2} \frac{1-e^{z}}{\varepsilon}+\ldots
$$

Example 2. Consider the Cauchy problem:

$$
\varepsilon \frac{d w}{d z}=e^{-w e^{z}}-10, \quad w(0, \varepsilon)=0 .
$$

Then

$$
w(z, \varepsilon)=e^{-z} \ln \frac{1+9 e^{10\left(1-e^{z}\right) / \varepsilon}}{10}+\varepsilon \frac{e^{-2 z}}{10} \ln \frac{1+9 e^{10\left(1-e^{z}\right) / \varepsilon}}{10}+\ldots
$$

is the sought solution pseudo-holomorphic in the global sense.

\section{HolOMORPHic REgUlarization OF TikHONOV SYSTEM}

We proceed to a real domain and in the segment $[0, T]$ we consider the initial problem for Tikhonov system with one slow and one fast variable:

$$
\begin{cases}\frac{d y}{d t}=f(t, y, v), & \varepsilon \frac{d v}{d t}=F(t, y, v), \\ y(0, \varepsilon)=y_{0}, & v(0, \varepsilon)=v_{0} .\end{cases}
$$

Here $\varepsilon>0$ is a small parameter and $y_{0}$ and $v_{0}$ are independent of this parameter. If we let $\varepsilon=0$, we obtain the degenerate system

$$
\left\{\begin{array}{l}
\frac{d \bar{y}}{d t}=f(t, \bar{y}, v) \\
0=F(t, \bar{y}, v),
\end{array}\right.
$$

for which we impose only the first initial condition $\bar{y}(0)=y_{0}$.

Let $v=\Phi(t, \bar{y})$ be a solution to the second equation in system (10), then we have the Cauchy problem:

$$
\frac{d \bar{y}}{d t}=f(t, \bar{y}, \Phi(t, \bar{y})), \quad \bar{y}(0)=y_{0} .
$$

Under the assumptions of Tikhonov theorem [1], the passage to limit holds:

$$
\begin{aligned}
\lim _{\varepsilon \rightarrow+0} y(t, \varepsilon) & =\bar{y}(t), & & 0 \leqslant t \leqslant T, \\
\lim _{\varepsilon \rightarrow+0} v(t, \varepsilon) & =\Phi(t, \bar{y}(t)), & & 0<t \leqslant T .
\end{aligned}
$$

Let us present the formalism of the method of holomorphic regularization for this system. Assume that its right hand sides are holomorphic in a closed domain $\bar{D}$ of the space of real variables $(t, y, v)$ containing the point $Q_{0}\left(0, y_{0}, v_{0}\right)$ and let $F(t, y, v) \neq 0$ in this domain. 
According the algorithm of the method, from nonlinear system (9) we proceed to the linear equation for its integrals:

$$
\varepsilon L U+F \frac{\partial U}{\partial v}=0
$$

where

$$
L=\frac{\partial}{\partial t}+f \frac{\partial}{\partial y}
$$

Assuming that the operator $L$ is relatively bounded with respect to the operator $F \frac{\partial}{\partial v}$, we seek a solution to equation (12) as a regular series in powers of $\varepsilon$ :

$$
U(t, y, v, \varepsilon)=U_{0}(t, y, v)+\varepsilon U_{1}(t, y, v)+\ldots+\varepsilon^{n} U_{n}(t, y, v)+\ldots,
$$

and for its coefficients we have a series of problems:

$$
\begin{aligned}
F \frac{\partial U_{0}}{\partial v} & =0, \\
F \frac{\partial U_{1}}{\partial v} & =-L U_{0}, \\
F \frac{\partial U_{2}}{\partial v} & =-L U_{1}, \\
& \cdots \\
F \frac{\partial U_{n}}{\partial v} & =-L U_{n-1},
\end{aligned}
$$

As a solution to the first equation in (14) we take an arbitrary holomorphic function $U_{0}=$ $\psi(t, y)$ independent of $v$.

Solving other equations under the condition $U_{n}\left(t, y, v_{0}\right)=0, n=1,2, \ldots$, we obtain

$$
U(t, y, v, \varepsilon)=\psi(t, y)-\varepsilon \int_{v_{0}}^{v} \frac{L \psi d v_{1}}{F\left(t, y, v_{1}\right)}+\varepsilon^{2} \int_{v_{0}}^{v}\left(L \int_{v_{0}}^{v_{1}} \frac{L \psi d v_{2}}{F\left(t, y, v_{2}\right)}\right) \frac{d v_{1}}{F\left(t, y, v_{1}\right)}-\ldots
$$

The proof of the convergence of series of form (15) can be found in [6]. It is easy to confirm that identity (15) defines a linear mapping $H_{F}^{\varepsilon}$ of the algebra $\mathcal{A}_{t y}$ of the functions holomorphic on the projection of the domain $\bar{D}$ on the space of the variables $(t, y)$, into the algebra $\mathcal{A}_{t y v}$ of the functions holomorphic on $\bar{D}$. Thus, $H_{F}^{\varepsilon}: \mathcal{A}_{t y} \rightarrow \mathcal{A}_{t y v}$ and $U(t, y, v, \varepsilon)=H_{F}^{\varepsilon}[\psi]$.

If we let $\psi=t$ and then $\psi=y$, we obtain two independent first integrals of system (9): $H_{F}^{\varepsilon}[t]$ and $H_{F}^{\varepsilon}[y]$. According the general theory of differential equations, there exists a function $\Psi$ such that $H_{F}^{\varepsilon}[\psi]=\Psi\left(H_{F}^{\varepsilon}[t], H_{F}^{\varepsilon}[y]\right)$. In this identity we let $v=v_{0}$, then $\psi=\Psi$, and this identity becomes a commutation relation:

$$
H_{F}^{\varepsilon}[\psi(t, y)]=\psi\left(H_{F}^{\varepsilon}[t], H_{F}^{\varepsilon}[y]\right) .
$$

Let us prove that the mappings $H_{F}^{\varepsilon}: \mathcal{A}_{t y} \rightarrow \mathcal{A}_{t y v}$ are homomorphisms. Indeed, for each $\psi_{1}, \psi_{2} \in \mathcal{A}_{t y}$ the identities

$$
H_{F}^{\varepsilon}\left[\psi_{1} \psi_{2}\right]=\left(\psi_{1} \psi_{2}\right)\left(H_{F}^{\varepsilon}[t], H_{F}^{\varepsilon}[y]\right)=\psi_{1}\left(H_{F}^{\varepsilon}[t], H_{F}^{\varepsilon}[y]\right) \psi_{2}\left(H_{F}^{\varepsilon}[t], H_{F}^{\varepsilon}[y]\right)=H_{F}^{\varepsilon}\left[\psi_{1}\right] H_{F}^{\varepsilon}\left[\psi_{2}\right] .
$$

hold true. Finally, the following theorem is true.

Theorem 3. To each system of form (9) there corresponds a holomorphic at the point $\varepsilon=0$ family $\left\{H_{F}^{\varepsilon}\right\}$ of homomorphisms of the algebra $\mathcal{A}_{t y}$ into the algebra $\mathcal{A}_{\text {tyv }}$ satisfying commutation relation (16); these homomorphisms are given by identity (15). The images of these homomorphisms are the integrals $U(t, y, v, \varepsilon)$ of this system holomorphic at the point $\varepsilon=0$. 
Let us introduce the notion of a pseudo-holomorphic solution to second order Tikhonov system [7].

Definition 2. A solution $(y(t, \varepsilon), v(t, \varepsilon))$ to initial problem (9) is called pseudo-holomorphic at the point $\varepsilon=0$ if there exist holomorphic at the point $M_{0}(0,0,0)$ functions $Y(t, \eta, \varepsilon)$ and $V(t, \eta, \varepsilon)$ such that for each $\varepsilon \in\left(0, \varepsilon_{0}\right)$, where $\varepsilon_{0}$ is some small positive number, there exists $\delta>0$ such that for all $t \in[0, \delta)$ the identities

$$
y(t, \varepsilon)=Y\left(t, \frac{\varphi(t)}{\varepsilon}, \varepsilon\right), \quad v(t, \varepsilon)=V\left(t, \frac{\varphi(t)}{\varepsilon}, \varepsilon\right)
$$

hold for some holomorphic on the segment $[0, T]$ function $\varphi(t)$; at that, $\varphi(0)=0$ and $\varphi^{\prime}(t)<0$ as $t \in[0, T]$.

If the series

$$
\sum_{n=0}^{\infty} Y_{n}(t, \eta) \varepsilon^{n}, \quad \sum_{n=0}^{\infty} V_{n}(t, \eta) \varepsilon^{n}
$$

representing the mentioned functions converge uniformly on the segment $[0, T]$ for each fixed $\eta \in$ $(-\infty ; 0]$ in some neighbourhood of the point $\varepsilon=0$ (depending on $\eta)$, the solution $(y(t, \varepsilon), v(t, \varepsilon)$ ) is called pseudo-holomorphic in the global sense.

The next theorem provides sufficient conditions for global pseudo-holomorphy.

Theorem 4. Under the assumptions of Tikhonov theorem on passage to the limit, the solution $(y(t, \varepsilon), v(t, \varepsilon))$ is pseudo-holomorphic at the point $\varepsilon=0$ in the global sense.

Proof. Let $\varphi(t)$ be a holomorphic on the segment $[0, T]$ function such that $\varphi(0)=0, \varphi^{\prime}(t)<0$ for all $t \in[0, T]$ and $\bar{y}(t)$ is a solution to Cauchy problem (11). We have to independent first integrals obtained from 15 as $\psi(t, y)=\varphi(t)$ and $\psi(t, y)=y-\bar{y}(t)$; these integrals define implicitly a solution $(y(t, \varepsilon), v(t, \varepsilon))$ :

$$
\begin{aligned}
& \int_{v_{0}}^{v} \frac{L \varphi d v_{1}}{F\left(t, y, v_{1}\right)}-\varepsilon \int_{v_{0}}^{v}\left(L \int_{v_{0}}^{v_{1}} \frac{L \varphi d v_{2}}{F\left(t, y, v_{2}\right)}\right) \frac{d v_{1}}{F\left(t, y, v_{1}\right)}+\ldots=\frac{\varphi(t)}{\varepsilon} \\
& y-\bar{y}(t)-\varepsilon \int_{v_{0}}^{v} \frac{L(y-\bar{y}(t)) d v_{1}}{F\left(t, y, v_{1}\right)}+\varepsilon^{2} \int_{v_{0}}^{v}\left(L \int_{v_{0}}^{v_{1}} \frac{L(y-\bar{y}(t)) d v_{2}}{F\left(t, y, v_{2}\right)}\right) \frac{d v_{1}}{F\left(t, y, v_{1}\right)}-\ldots=0 .
\end{aligned}
$$

In view of the fact that $L \varphi=\varphi^{\prime}(t)$, we write these equations as a system denoting the right hand side in (17) by $\eta$ :

$$
\left\{\begin{array}{l}
\varphi^{\prime}(t) \int_{v_{0}}^{v} \frac{d v_{1}}{F\left(t, y, v_{1}\right)}-\varepsilon \int_{v_{0}}^{v}\left(L \int_{v_{0}}^{v_{1}} \frac{\varphi^{\prime}(t) d v_{2}}{F\left(t, y, v_{2}\right)}\right) \frac{d v_{1}}{F\left(t, y, v_{1}\right)}+\ldots=\eta \\
y-\bar{y}(t)-\varepsilon \int_{v_{0}}^{v} \frac{-\bar{y}^{\prime}(t)+f\left(t, y, v_{1}\right)}{F\left(t, y, v_{1}\right)} d v_{1} \\
+\varepsilon^{2} \int_{v_{0}}^{v}\left(L \int_{v_{0}}^{v_{1}} \frac{-\bar{y}^{\prime}(t)+f\left(t, y, v_{1}\right)}{F\left(t, y, v_{2}\right)} d v_{2}\right) \frac{d v_{1}}{F\left(t, y, v_{1}\right)}-\ldots=0 .
\end{array}\right.
$$

Then we apply the implicit function theorem and the proof is completed in the same way as for Theorem 2. At that we employ the fact that the boundedness of the function $v=V_{0}(t, \varphi(t) / \varepsilon)$ 
as $\varepsilon \rightarrow+0$ uniformly in $t \in[0, T]$ defined by the equation

$$
\varphi^{\prime}(t) \int_{v_{0}}^{v} \frac{d v_{1}}{F\left(t, \bar{y}(t), v_{1}\right)}=\frac{\varphi(t)}{\varepsilon}
$$

is due to the asymptotic stability of the equilibrium $\widetilde{v}=\Phi(t, y)$ for so-called adjoint equation

$$
\frac{d \widetilde{v}}{d \tau}=F(t, y, \widetilde{v}), \quad \tau \geq 0,
$$

that is Condition IV of Tikhonov theorem on passage to the limit [1]. The proof is complete.

Remark. In various asymptotic methods, a choice of the regularizing function $\varphi(t)$ is made in different ways. In Vasilieva-Butuzov-Nefedov method the choice is $\varphi(t)=-t$; in Lomov regularization method the choice is determined by the spectrum of some operator related with a considered singularly perturbed problem. A quite effective method for studying nonlinear problem is Safonov method of normal forms [4]. In most cases both methods give rise to an exponential boundary layer.

Concerning the method of holomorphic regularization, here the following statement holds: if the equation

$$
\varphi^{\prime}(t) \int_{v_{0}}^{v} \frac{d v_{1}}{F\left(t, \bar{y}(t), v_{1}\right)}=\eta
$$

has a solution of form $v=V_{0}\left(t, e^{\eta}\right)$, where the function $V_{0}(t, q)$ is holomorphic in the rectangle $\Pi_{t q}=\{(t, q): 0 \leqslant t \leqslant T, 0 \leqslant q \leqslant 1\}$, then solution to initial problem (9) is pseudoholomorphic on the segment $[0, T]$ in the global sense.

We write formulae of first order in $\varepsilon$ :

$$
\begin{aligned}
y(t, \varepsilon)= & \bar{y}(t)+\left.\varepsilon \int_{v_{0}}^{v} \frac{L(y-\bar{y}(t)) d v_{1}}{F\left(t, y, v_{1}\right)}\right|_{\substack{y=\bar{y}(t) \\
v=V_{0}(t, \varphi(t) / \varepsilon)}}+\overline{\bar{o}}(\varepsilon), \quad \varepsilon \rightarrow 0 ; \\
v(t, \varepsilon)= & V_{0}\left(t, \frac{\varphi(t)}{\varepsilon}\right)+\varepsilon \frac{F(t, y, v)}{\varphi^{\prime}(t)}\left[\int_{v_{0}}^{v}\left(L \int_{v_{0}}^{v_{1}} \frac{\varphi^{\prime}(t) d v_{2}}{F\left(t, y, v_{2}\right)}\right) \frac{d v_{1}}{F\left(t, y, v_{1}\right)}\right. \\
& \left.-\int_{v_{0}}^{v} \frac{L(y-\bar{y}(t)) d v_{1}}{F\left(t, y, v_{1}\right)} \cdot \frac{\partial}{\partial y} \int_{v_{0}}^{v} \frac{\varphi^{\prime}(t) d v_{1}}{F\left(t, y, v_{1}\right)}\right]\left.\right|_{\substack{y=\bar{y}(t) \\
v=V_{0}(t, \varphi(t) / \varepsilon)}}+\overline{\bar{o}}(\varepsilon), \quad \varepsilon \rightarrow 0 .
\end{aligned}
$$

Example 3. We consider the Cauchy problem

$$
\begin{cases}\frac{d y}{d t}=v^{2}, & \varepsilon \frac{d v}{d t}=v^{2}-y^{2} e^{2 t}, \quad t \in[0, T], \quad \varepsilon>0 \\ y(0, \varepsilon)=-2, & v(0, \varepsilon)=0 .\end{cases}
$$

The proposed approach gives:

$$
\begin{aligned}
& y(t, \varepsilon)=-2 e^{-2 t}-2 \varepsilon e^{-t} \tanh \frac{2\left(e^{-t}-1\right)}{\varepsilon}+\overline{\bar{o}}(\varepsilon), \quad \varepsilon \rightarrow 0, \\
& v(t, \varepsilon)=2 e^{-t} \tanh \frac{2\left(e^{-t}-1\right)}{\varepsilon}+\overline{\bar{o}}(1), \quad \varepsilon \rightarrow 0 .
\end{aligned}
$$




\section{CONCLUSION}

In theoretical physics, a so-called Dyson's argument is known [8]. It expresses the property of non-analiticity for solution to each (in the general case) singularly perturbed problem and following Poincaré theorem on expansion. In particular, in non-relativistic gravitation theory, while solving problem on hydrostatic equilibrium of stars without local electroneutrality constraint, the expansion is made in the gravitation constant $G$ in the vicinity of the point $G=0$. In the present work we provide conditions ensuring the pseudo-holomorphy and hence, there arises a chance to construct the solutions to singularly perturbed problems as power series in the small parameter converging in the usual sense.

\section{BIBLIOGRAPHY}

1. A.B. Vasil'eva, V.F. Butuzov. Asymptotic expansion of solutions for singularly perturbed equations, Nauka, Moscow (1973). (in Russian).

2. S.A. Lomov. Introduction to the general theory of singular perturbations. Nauka, Moscow (1981). [Transl. Math. Monog. 112. Amer. Math. Soc. Providence, RI (1992).]

3. S.A. Lomov, I.S. Lomov. Foundations of mathemathical theory of boundary layer. Moscow State Univ. Publ., Moscow (2011). (in Russian).

4. V.F. Safonov. Nonlinear regularization of singularly perturbed resonance problems and analyticity of their solutions in the perturbation parameter // Sibir. Matem. Zhurn. 33:6, 178-187 (1992). [Siber. Math. J. 33:6, 1109-1118 (1992).]

5. V.I. Kachalov. Holomorphic regularization of singularly perturbed problems // MPEI Vestnik. 6, 54-62 (2010). (in Russian).

6. V.I. Kachalov. Holomorphic in parameter integrals of singularly perturbed systems MPEI Vestnik. 6, 57-68 (2011). (in Russian).

7. V.I. Kachalov. Tikhonov's theorem on passage to the limit and pseudoholomorphic solutions of singularly perturbed problems // Dokl. RAN. 458:6, 630-632 (2014). [Dokl. Math. 90:2, 616-618 (2014).]

8. M.I. Krivoruchenko, D.K. Nadyozhin, A.V. Yudin. Hydrostatic equilibrium of stars without electroneutrality constraint// Phys. Rev. D. 97:15, id 083016 (2018).

Vasilii Ivanovich Kachalov,

National Research University "MPEI",

Krasnokazarmennaya str. 14,

111250, Moscow, Russia

E-mail: vikachalov@rambler.ru 